\title{
UMA ABORDAGEM DIDÁTICA DA MODELAGEM DE UMA PLANTA DE NÍVEL UTILIZANDO FACTORY IO, CODESYS E LABVIEW
}

DOI: 10.37702/2175-957X.COBENGE.2021.3476

Pablo France Salarolli - pablo_salarolli@hotmail.com

Instituto Federal do Espírito Santo

Av Des Antonio Jose M Feu Rosa 647

29172-680 - Serra - ES

Luiza Broseghini Pin - luizabrpin@gmail.com

Instituto Federal do Espírito Santo

rua primeiro de maio 188

29120-260 - vila velha - ES

Flávio Garcia Pereira - flavio.garcia@ifes.edu.br

Instituto Federal do Espírito Santo

Rodovia ES $010 \mathrm{~km} \mathrm{6,5}$

29173-087 - Serra - ES

Wagner Teixeira da Costa - wagnercosta@ifes.edu.br

Instituto Federal do Espírito Santo

Rodovia ES-010 km 6,5

29173-087 - Serra - ES

Marco Antonio de Souza Leite Cuadros - marcoantonio@ifes.edu.br Instituto Federal do Espírito Santo

Av. Copacabana 569

29166-820 - Serra - ES

Rafael Peixoto Derenzi Vivacqua - rafsatpdv@gmail.com

Instituto Federal do Espírito Santo

Avenida Dos Sabiás 182

29166-630 - Serra - ES

Resumo: As áreas de automação, controle, modelagem de sistema e otimização possuem fundamental contribuição para a melhoria dos processos utilizados pelas indústrias, proporcionando um processo de produção seguro e rápido, aprimorando 
a qualidade do produto final e reduzindo os custos de produção. Boas ferramentas para o desenvolvimento de pesquisas nestas áreas são os softwares de simulações didáticos, pois proporcionam um espaço seguro para experimentar com problemas reais do setor industrial. Em vista dessas capacidades, o objetivo deste trabalho é apresentar uma metodologia didática de modelagem de uma planta de nível simulada em um ambiente 3D. Foram utilizados os softwares Factory 10, Codesys e LabVIEW trabalhando em conjunto. Dessa forma, não é necessário possuir uma planta real em um laboratório, o que muitas vezes implica em investimentos financeiros. Também é uma alternativa para os trabalhos das disciplinas que estão sendo ofertadas à distância.

Palavras-chave: Modelagem de sistemas, Simulação, Sistemas de controle 


\section{UMA ABORDAGEM DIDÁTICA DA MODELAGEM DE UMA PLANTA DE NÍVEL UTILIZANDO FACTORY IO, CODESYS E LABVIEW}

\section{INTRODUÇÃO}

A modelagem de sistemas nos institutos de ensino, tem surgido com o intuito de estimular a capacidade do aluno a desenvolver soluções para problemas reais no setor industrial. Esses sistemas possibilitam a criação e simulações em ambientes controlados, permitindo, em conjunto com a fundamentação teórica apresentada em sala de aula, o desenvolvimento de soluções para situações e problemas reais existentes no setor industrial (FONSECA, 2012).

Vários trabalhos científicos têm utilizado plantas pilotos ou didáticas. Os trabalhos de MARTIN (2006), OLIVEIRA (2008) e THOMAS et al. (2010) abordam metodologias de controle de processo como, modelagem, identificação e controle de sistemas.

O presente trabalho realiza experimentos em uma planta de nível, usando softwares de simulação didáticos. Será apresentado desde o modelo matemático do processo até a metodologia utilizada para a obtenção do modelo experimental da planta, permitindo avaliar seu funcionamento antes de a sua aplicação no processo real.

\section{AMBIENTE DE SIMULAÇÃO}

\subsection{Estrutura utilizada}

Neste trabalho foram utilizados os softwares: Factory I/O, como ambiente de simulação; Codesys, como meio de comunicação entre os softwares; e LabVIEW, para controle e aquisição de dados, a fim de obter a modelagem de uma planta de nível.

\subsection{Factory 10}

O Facory I/O é um programa desenvolvido pela Real Games, e consiste em um ambiente de simulação 3D de fábricas, com o intuito de facilitar o ensino de tecnologias de automação. Esse software oferece a possibilidade de criarmos as nossas próprias cenas, simulando os sistemas que encontramos na indústria. É possível controlar os processos criados no Factory IO de múltiplas formas, aumentando a versatilidade na escolha da tecnologia a ser implementada (FACTORY IO, 2021; SÁNCHEZ, 2018). Neste trabalho o Facotry IO é utilizado para a simular a planta de nível em um ambiente 3D.

\subsection{LabVIEW}

O LabVIEW é um ambiente de programação gráfica, desenvolvido pela National Instruments e é utilizado no desenvolvimento de sistemas de medição, teste, controle e automação. O funcionamento do código em LabVIEW segue o modelo de fluxo de dados, e sua programação é feita utilizando ícones gráficos e ligações entre estes ícones (EGLES, 2017). Dentro do escopo do trabalho, o LabVIEW é encarregado de realizar todo o controle de malha aberta da planta e a coleta e salvamento dos dados em arquivo, para a posteriormente obter os modelos do processo. O LabVIEW foi escolhido para essa tarefa 
devido sua interface amigável, facilidade de integração e de criar programas que interajam com o usuário e de gerar arquivos com os dados coletados nos testes.

\subsection{Codesys}

O Codesys é uma plataforma de desenvolvimento de aplicações para controladores programáveis, e possui uma ampla gama de aplicações industriais. Nesse programa tudo está integrado em uma única interface de usuário (CODESYS, 2021). Nele é possível criar e executar um código para um controlador lógico programável virtual, controlando as variáveis das plantas do Factory IO através da comunicação OPC. No presente trabalho, o Codesys foi utilizado como um servidor OPC, onde as variáveis utilizadas no sistema foram configuradas nele, e assim a comunicação entre o Factory IO e o LabVIEW foi estabelecida. Numa aplicação de teste de controladores, o Codesys pode ser utilizado como o elemento controlador, e o LabVIEW como sistema supervisório, o que simula uma arquitetura muito utilizada no ambiente industrial.

\section{MODELAGEM}

\subsection{A planta}

A planta utilizada no Factory 10 pode ser vista na Figura 1 e consiste em um tanque com duas válvulas de controle: uma de saída e uma de entrada, ligadas às partes superior e inferior do tanque, respectivamente. Essas válvulas são atuadas por sinal analógico de tensão com faixa de 0 a $10 \mathrm{~V}$. No tanque há também um sensor de nível, que fornece um sinal analógico de 0 a $10 \mathrm{~V}$, abrangendo todos os valores de nível possíveis do tanque. Foi montado também um painel com algumas botoeiras e sinalizadores, para simular uma operação manual, porém, todo controle de abertura e fechamento das válvulas foi feito utilizando o LabVIEW.

Figura 1 - Planta utilizada no Factory IO.

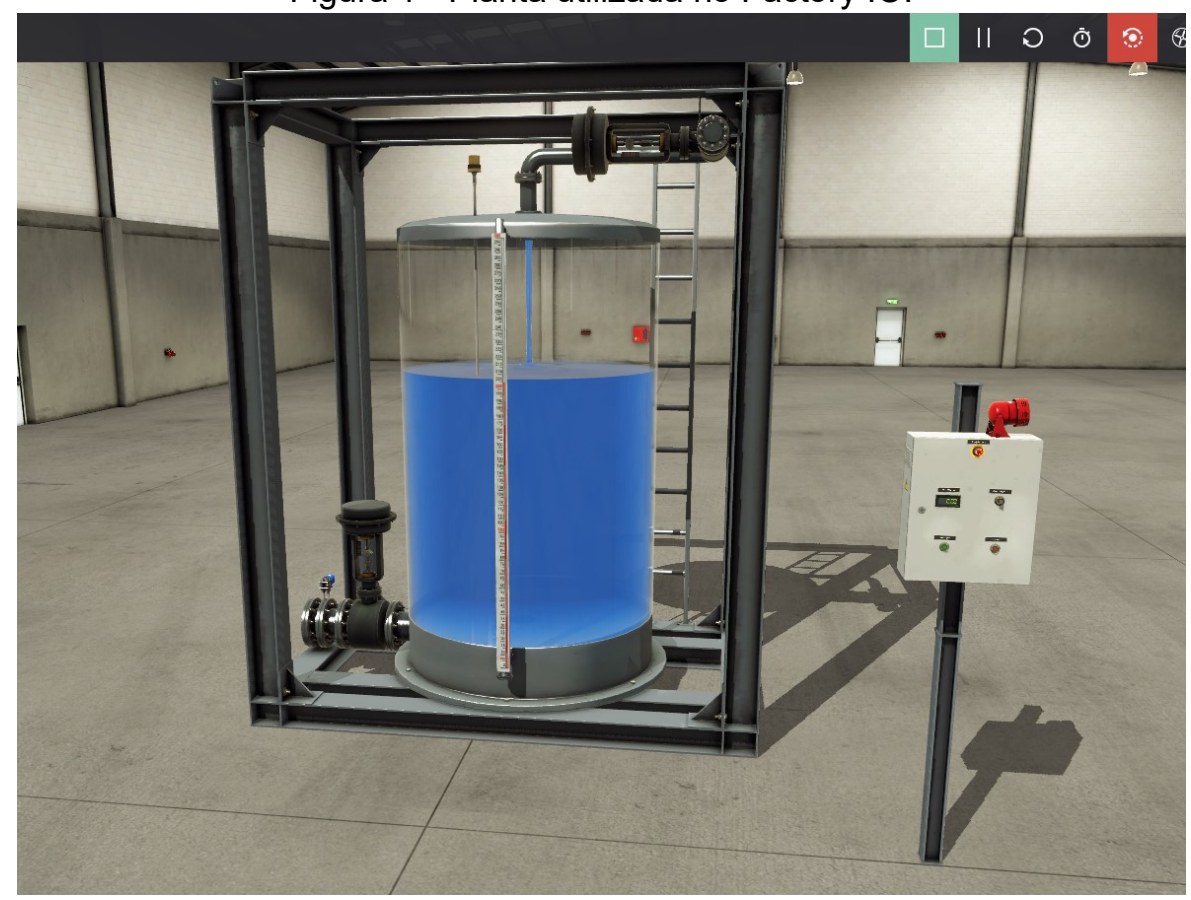

Fonte: Próprio Autor (2021). 
Para modelar esta planta, será utilizada como princípio básico a lei da conservação da massa, e algumas simplificações e aproximações serão consideradas: o fluido utilizado é incompressível com massa específica constante, o tanque possui seção uniforme de área constante, as resistências fluídicas são constantes (TAVARES, 2017).

Ao modelar a planta, o objetivo é chegar em uma função de transferência similar a Equação (1), onde é descrita a relação entre a altura do tanque $H(s)$ e a vazão de entrada $Q_{i}(s)$. Essa é uma função transferência de primeira ordem, que possui os parâmetros ganho $(K)$ e constante de tempo $(\tau)$. O ganho é o fator que relaciona a amplitude da variação na saída do sistema dada uma variação na entrada. Já a constante de tempo, indica o quão rápido o sistema retorna ao regime permanente, após uma variação na entrada.

$$
G(s)=\frac{H(s)}{Q_{i}(s)}=\frac{K}{\tau s+1}
$$

A demonstração a seguir é baseada no livro de OGATA (2010). A planta pode ser representada pelo diagrama da Figura 2, onde as variáveis $\bar{Q}$ e $\bar{H}$ são, respectivamente vazão e nível do fluido em regime permanente, enquanto as variáveis $q_{i}, q_{o}$ e $h$ são, respectivamente, pequenas variações na vazão de entrada, na vazão de saída e no nível do tanque. As variáveis $R$ e $C$, representam, respectivamente, a resistência ao fluxo de líquido na tubulação de saída e a capacitância do tanque (relação entre a variação do volume de líquido com a variação no nível do tanque).

Figura 2 - Diagrama da planta de nível.

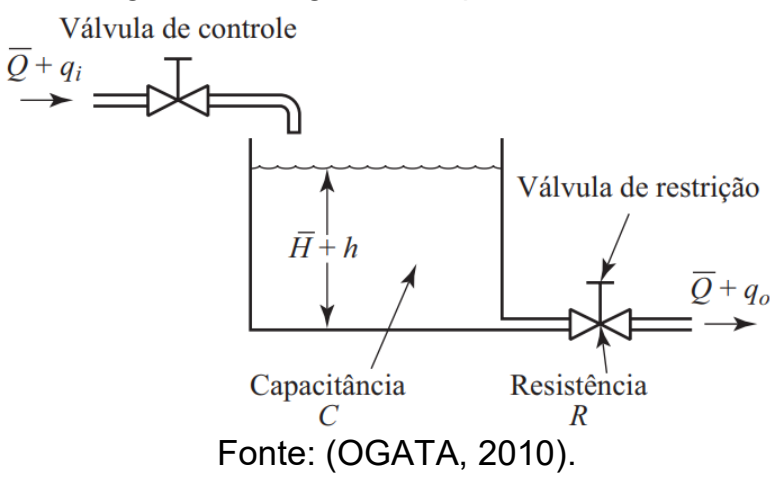

Como a relação entre a vazão de saída e o nível do tanque é uma função não linear $(Q=K \times \operatorname{sqrt}(H))$, conforme pode ser visto na curva presente na

Figura 3, trata-se de um sistema não linear. Porém, para pequenas variações em torno de um ponto de operação, é possível linearizar o sistema, utilizando uma aproximação (OGATA, 2010), que pode ser vista na Equação (4).

Figura 3 - Relação entre taxa de escoamento e altura da coluna de líquido do tanque. 


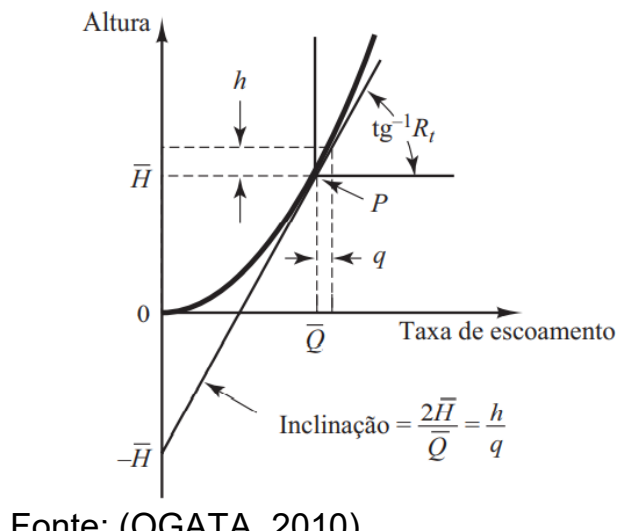

Fonte: (OGATA, 2010).

A variação do volume de fluido no tanque pode ser representada pela Equação (2).

$$
\frac{d V}{d t}=q_{i}(t)-q_{o}(t)
$$

Pela definição de capacitância e pela simplificação aproximada na fórmula da resistência, temos as Equações (3) e (4):

$$
\begin{gathered}
C=\frac{d V}{d h} \\
R=\frac{h}{q_{o}}
\end{gathered}
$$

Substituindo essas duas definições na Equação (2), temos a Equação (5):

$$
R C \frac{d h}{d t}+h=R q_{i}
$$

Aplicando a transformada de Laplace na equação acima(5), temos a Equação (6), que ao ser rearranjada, é possível obter a função de transferência da Equação (7):

$$
\begin{gathered}
\mathcal{L}(h)=H(s), \mathcal{L}\left(q_{i}\right)=Q_{i}(s) \rightarrow(R C s+1) H(s)=R Q_{i}(s) \\
G(s)=\frac{H(s)}{Q_{i}(s)}=\frac{R}{R C s+1}
\end{gathered}
$$

\subsection{Metodologia de modelagem}

Para obter o modelo da planta, foi utilizada a técnica de análise da resposta de testes ao degrau. Para obter o modelo, foram executadas três etapas:

\section{Ajustes na planta}

Inicialmente, foi definido quão aberta a válvula de saída do tanque estaria durante a operação. Esta etapa é importante, pois caso a válvula de saída fique toda fechada, a planta se comportará de forma completamente diferente (como se fosse um integrador), com o nível apenas aumentando, impossibilitando o uso de um modelo de primeira ordem. E caso a válvula esteja completamente aberta, é possível que a faixa de níveis de fluido no tanque 
que se alcance seja limitada, devido à grande vazão de saída. Foi definido o valor de $45 \%$ da faixa, que equivale a $4,5 \mathrm{~V}$.

\section{Definição do ponto de operação}

Como foi apresentado acima, o sistema que está sendo modelado é não linear e para utilizar as aproximações e linearizações que permitem considerá-lo como um sistema linear de primeira ordem é necessário obedecer à restrição de utilizar um ponto de operação e que as variações em torno desse ponto sejam pequenas.

Para tanto, foram aplicados degraus de $5 \%$ da faixa da válvula de entrada, varrendo toda a faixa, de 0 a $10 \mathrm{~V}$ e retornando a 0 , totalizando 40 degraus. Em todo degrau aplicado, aguardou-se o sistema entrar em regime permanente antes de aplicar o degrau subsequente. Este teste foi realizado com o intuito de analisar o comportamento da planta ao longo de toda sua faixa.

A Figura 4 contém as respostas aos degraus de subida. Nota-se no fim dos testes uma área destacada em vermelho, os dados desta região foram descartados pois o tanque chegou ao seu nível máximo, saturando o elemento sensor. A Figura 5 é análoga à Figura 4 porém, contendo as respostas aos degraus de descida.

Figura 4 - Testes à degraus de subida ao longo de toda faixa de operação.

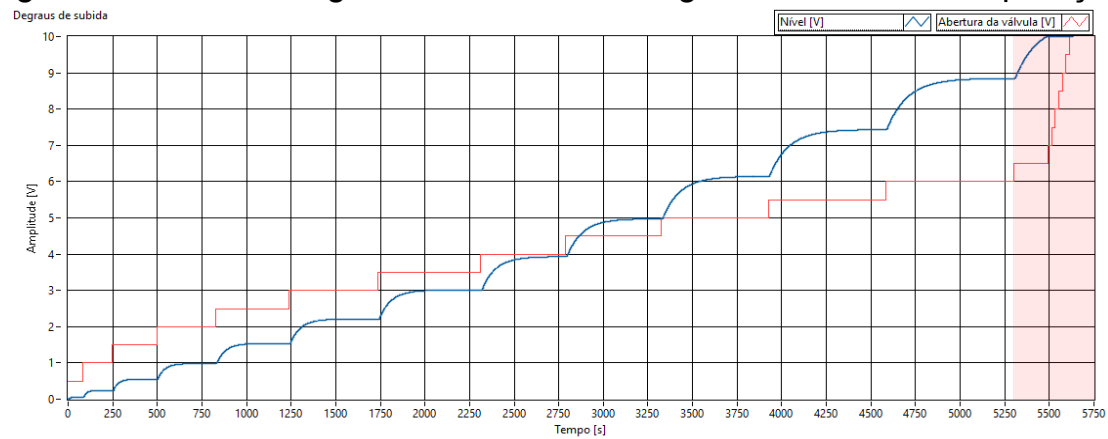

Fonte: Próprio Autor (2021).

Figura 5 - Testes à degraus de descida ao longo de toda faixa de operação.

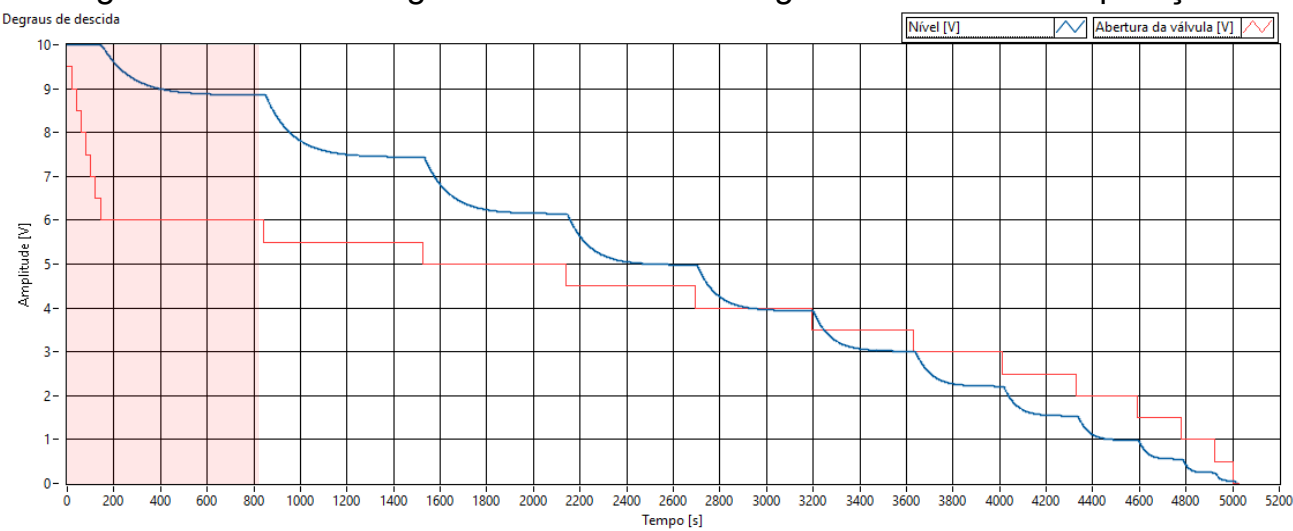

Fonte: Próprio Autor (2021)

Com as curvas das figuras acima, foi possível calcular os valores de ganho e constante de tempo para cada degrau aplicado. Destaca-se aqui também a área em vermelho, onde os dados foram descartados, por estar no fim da faixa. Observando os dados obtidos, foi decidido trabalhar com o ponto de operação entre $45 \%$ e $50 \%$ da faixa da válvula de entrada, ou seja, entre 4,5 e $5 \mathrm{~V}$, por ser uma região próxima ao cento da faixa, proporcionando uma distância razoável da região onde o sensor fica saturado. 
Figura 6 - Comportamento do ganho e da constante de tempo com base nos testes ao longo de toda faixa de operação.

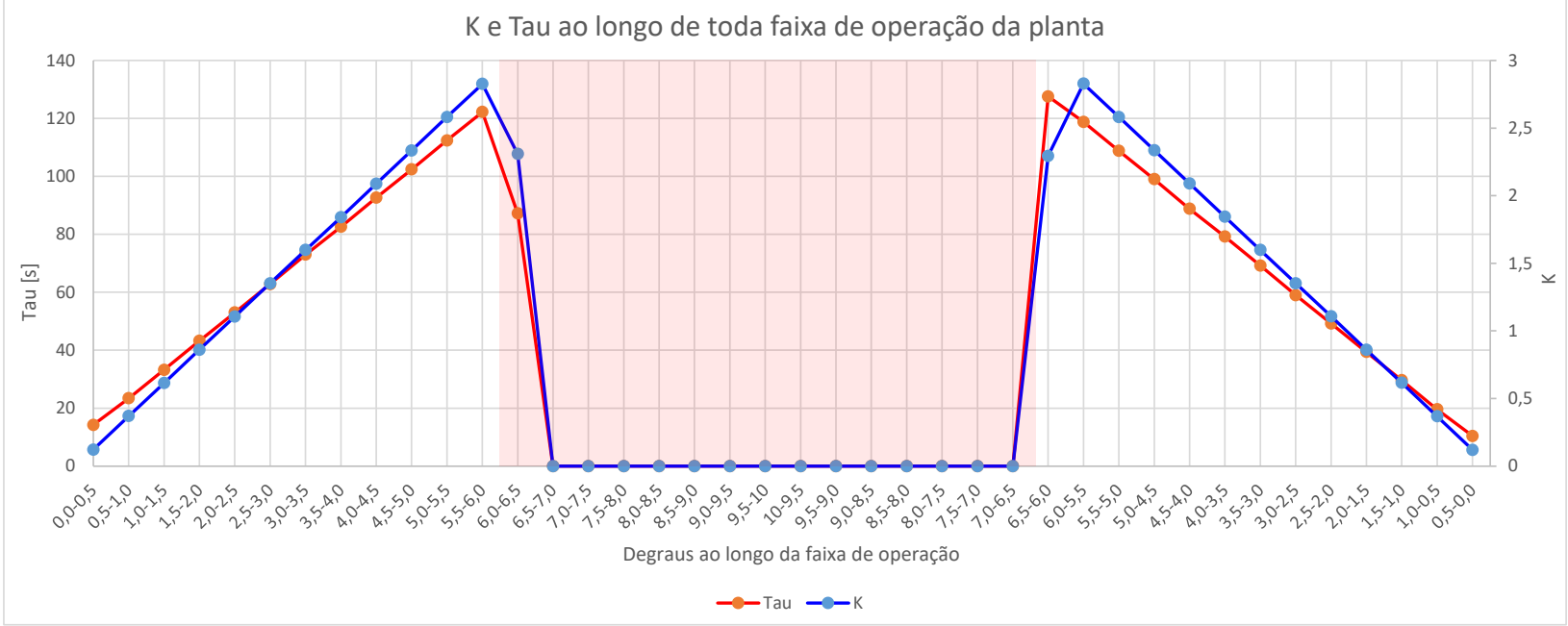

Fonte: Próprio Autor (2021).

\section{Obtenção do modelo}

Com a faixa de operação selecionada, seguiu-se a recomendação de CAMPOS; TEIXEIRA (2006) de aplicar ao menos três variações ao degrau, foram aplicados três degraus de subida e três degraus de descida dentro da faixa de operação selecionada. Após a obtenção dos parâmetros para os seis degraus, os valores finais do modelo equivalem a média aritmética dos valores obtidos. As curvas obtidas destes testes ao degrau podem ser vistas na Figura 7.

Figura 7 - Testes ao degrau para obtenção do modelo.

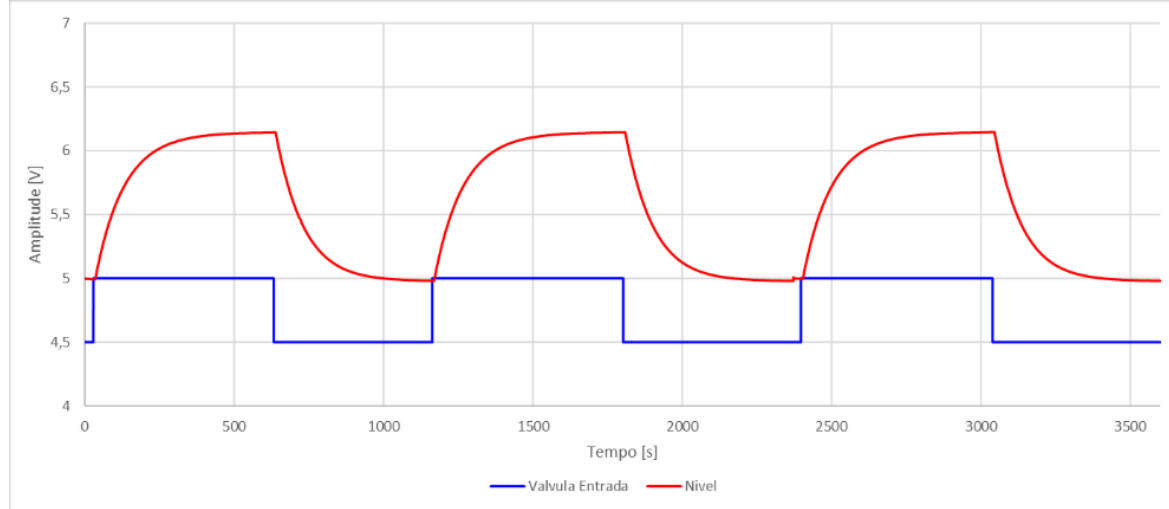

Fonte: Próprio Autor (2021).

O procedimento de obter o modelo a partir de dados experimentais de testes ao degrau em um sistema de primeira ordem com função de transferência da forma da Equação (1) consiste em obter os parâmetros ganho $(K)$ e constante de tempo $(\tau)$. A entrada do sistema consiste na variável que serão aplicados os degraus enquanto a saída consiste na variável medida que se deseja modelar. No caso do sistema em questão, a entrada $x$ é a tensão 
aplicada na válvula de entrada de líquido no tanque, enquanto a saída $y$ é a tensão fornecida pelo sensor de nível do tanque.

O ganho $K$ é a razão entre a variação da saída e a variação da entrada, conforme a Equação (8). Vale ressaltar que antes de se aplicar um degrau, é necessário que o sistema esteja em regime permanente. As entrada e saída iniciais são obtidas no gráfico no instante imediatamente anterior a aplicação do degrau que está sendo analisado. Já as entrada e saída finais são obtidas no sinal após o sistema entrar novamente em regime permanente após a aplicação do degrau que está sendo analisado.

$$
K=\frac{\Delta y}{\Delta x}=\frac{y_{\text {final }}-y_{\text {inicial }}}{x_{\text {final }}-x_{\text {inicial }}}
$$

Já a constante de tempo $\tau$ é o tempo para que a saída do sistema alcance o valor de aproximadamente $63,2 \%$ de sua variação total $\Delta y$, conforme pode ser visto na Equação (9), onde $t_{0}$ é o instante onde inicia-se a variação na saída $y$. Para obter $\tau$, é necessário calcular o valor de $y\left(t_{0}+\tau\right)$, e analisar graficamente qual o instante equivalente a este valor calculado. Este valor é igual a $t_{0}+\tau$. Em seguida, deve-se analisar graficamente qual o valor de $t_{0}$, para enfim conseguir obter o valor da constante de tempo $\tau$.

$$
y\left(t_{0}+\tau\right)=y_{\text {inicial }}+0,632 \Delta y
$$

Durante os testes foi possível notar um pequeno atraso de transporte, no valor aproximado de 6,8 segundos e como o valor de constante de tempo é mais de 10 vezes maior que $o$ atraso de transporte, esse valor foi desprezado. Com as curvas, foi possível calcular os valores de ganho e constante de tempo para cada curva. Ao fim, foi calculada a média aritmética dos valores obtidos e estas médias foram tomados como os valores de ganho e constante de tempo da planta. Todos estes valores podem ser vistos na Tabela 1.

Tabela 1 - Resultados dos testes ao degrau.

\section{\begin{tabular}{|c|c|c|c|c|c|c|}
\hline Subida 1 & Descida 1 & Subida 2 & Descida 2 & Subida 3 & Descida 3 & Média \\
\hline
\end{tabular}}

\begin{tabular}{c|c|c|c|c|c|c|c|}
$\mathbf{K}$ & 2,302 & 2,324 & 2,326 & 2,328 & 2,302 & 2,330 & $\mathbf{2 , 3 1 9}$ \\
$\mathbf{T}$ & 100,60 & 99,00 & 102,80 & 99,60 & 101,00 & 99,00 & $\mathbf{1 0 0 , 3 3}$ \\
\hline
\end{tabular}

Fonte: Próprio Autor (2021).

Assim, a função de transferência obtida da planta foi:

$$
G(s)=\frac{2,319}{100,33 s+1}
$$

\section{RESULTADOS}

Como resultado, foram aplicados degraus no modelo obtido e as curvas foram comparadas às respostas reais da planta. Podemos ver esses comparativos as Figuras 8 e 9. Nota-se o pequeno atraso de transporte nas curvas referentes à planta, e que é válida a aproximação de ignorar este parâmetro, pois o modelo apresentou comportamento coerente ao longo de toda curva. 
Figura 8 - Comparativo entre o modelo e a planta para o degrau de descida.

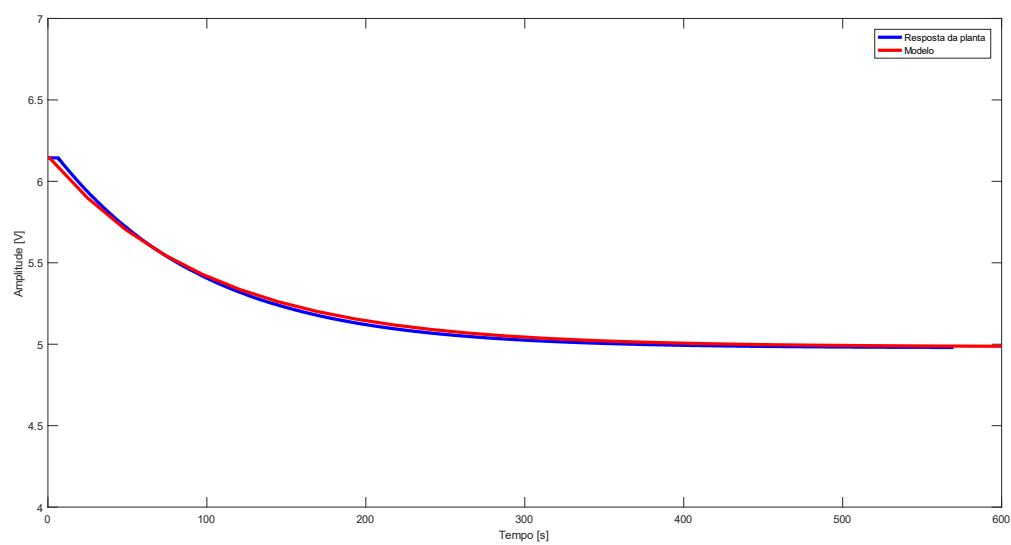

Fonte: Próprio Autor (2021).

Figura 9 - Comparativo entre o modelo e a planta para o degrau de subida.

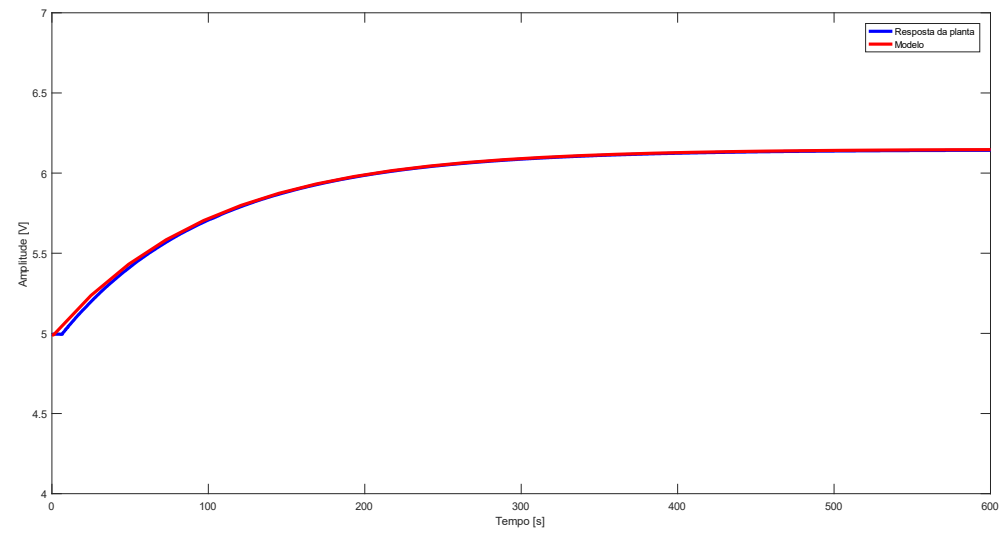

Fonte: Próprio Autor (2021)

Comparando a similaridade das curvas, é possível observar que o modelo obtido, mesmo ignorando o pequeno atraso de transporte, representa a planta do processo.

\section{CONCLUSÃO}

O trabalho apresentou uma metodologia de modelagem prática de um sistema de primeira ordem. Neste trabalho foram utilizadas ferramentas de simulação, o que simplifica os procedimentos para os alunos e professores. Porém, vale ressaltar que a metodologia apresentada também pode ser utilizada em uma planta real. Além do modelo prático, foi demonstrado como é obtido o modelo matemático do sistema em questão, pois a teoria e a prática são complementares no processo de aprendizado.

\section{Agradecimentos}

Agradecemos à FAPES (Fundação de Amparo à Pesquisa e Inovação do Espírito Santo) e a CAPES (Coordenação de Aperfeiçoamento de Pessoal de Nível Superior) pelo apoio financeiro dado por meio do PDPG (Parcerias Estratégicas nos Estados da CAPES).

\section{REFERÊNCIAS}


CAMPOS, M. C. M. M. de; TEIXEIRA, H. C. G. Controles típicos de equipamentos e processos industriais. São Paulo: Edgard Blucher, 2006. E-book.

CODESYS. Why CODESYS?. [s. I.], 2021. Disponível em: https://www.codesys.com/thesystem/why-codesys.html. Acesso em: 4 fev. 2021.

EGLES, J. M. Automação da medição de forças e vibrações no torneamento com plataforma em labview. 2017. - Universidade Tecnológica Federal do Paraná, [s. I.], 2017. Disponível em: http://repositorio.utfpr.edu.br:8080/jspui/handle/1/9500. Acesso em: 8 fev. 2021.

FACTORY IO. Factory I/O - Documentation. [s. I.], 2021. Disponível em: https://docs.factoryio.com/. Acesso em: 4 fev. 2021.

FONSECA, D. G. V. da. Modelagem e controle adaptativo de uma planta didática de nível com instrumentação industrial. 2012. - Universidade Federal do Rio Grande do Norte, [s. I.], 2012. Disponível em: https://repositorio.ufrn.br/handle/123456789/15446. Acesso em: 8 fev. 2021.

MARTIN, P. A. Modelagem e controle de um trocador de calor feixe tubular. 2006. Biblioteca Digital de Teses e Dissertações da Universidade de São Paulo, São Paulo, 2006. Disponível em: https://doi.org/10.11606/D.3.2006.tde-27032006-211526. Acesso em: 8 fev. 2021.

OGATA, K. Engenharia de Controle Moderno. [S. I.: s. n.]. E-book.

OLIVEIRA, E. M. Implementação de técnicas de controle avançado a uma planta piloto de controle de vazão e temperatura de ar. 2008. - Universidade Federal de Minas Gerais, [s. I.], 2008. Disponível em: https://repositorio.ufmg.br/handle/1843/FGJA7D6JRR. Acesso em: 8 fev. 2021.

SÁNCHEZ, J. M. Diseño de un sistema de control distribuido usando Factory IO y Codesys V3 (comunication through OPC). 2018. [s. I.], 2018. Disponível em: https://idus.us.es/handle/11441/83982. Acesso em: 8 fev. 2021.

TAVARES, M. F. Modelagem de sistemas dinâmicos. Londrina: Editora e Distribuidora Educacional S.A, 2017. E-book.

THOMAS, W. G. et al. Modelagem de uma planta industrial dldática multivariável não linear. In: 2010, 2010 9th IEEE/IAS International Conference on Industry Applications, INDUSCON 2010. [S. I.: s. n.] Disponível em: https://doi.org/10.1109/INDUSCON.2010.5739864

\section{A DIDACTIC APPROACH OF MODELING A LEVEL PROCESS USING FACTORY IO, CODESYS AND LABVIEW}


Abstract: The areas of automation, control, system modeling and optimization have a fundamental contribution to the improvement of the processes used by the industries, providing a safe and fast production process, improving the quality of the final product and reducing production costs. Good tools for the development of research in these areas are didactic simulation software, as they provide a real view of the industrial sector. The objective of this work is to present a didactic methodology for modeling a simulated level process in a $3 D$ environment. Factory IO, Codesys and LabVIEW software were used working together. Thus, it is not necessary to have a real plant in a laboratory, which often implies financial investments. It is also an alternative for the work of the disciplines that are being offered at a distance.

Keywords: Systems modeling, Simulation, Control system 